\title{
Modernization of Processes Control Methods for Digital Image Processing
}

\author{
Tashmanov E.B.
}

Tashkent University of Information Technologies, Uzbekistan

Copyright $\mathrm{C} 2016$ by authors, all rights reserved. Authors agree that this article remains permanently open access under the terms of the Creative Commons Attribution License 4.0 International License

\begin{abstract}
The article is devoted to studying the chasing problem games regarding to the levels of digital image brightness, described by discrete second order linear equations. We obtained sufficient conditions for finish of chase. Partly on a model example we showed that using the management in specified area, you could define a certain level of brightness in a digital image in case of presence of the player, which prevented this transition.
\end{abstract}

Keywords The Image, Chebyshev's Polynoms, Stirring Video Objects, Reconstruction of Images, Accuracy of Restoration of Pixels

\section{Introduction}

The model example of the control problems studied class is the following process of persecution brightness levels of digital image described by equations ([1]-[3])

$$
\begin{gathered}
-4 z_{i, j}+z_{i-1, j}+z_{i+1, j}+z_{i, j-1}+z_{i, j+1} \\
=-u_{i, j}+v_{i, j},\left|u_{i, j}\right| \leq \rho,\left|v_{i, j}\right| \leq \sigma, \sigma<\rho, \\
z_{0, j}=0, z_{\mathrm{m}+1, j}=0, \quad z_{i, 0}=0, z_{i, \theta}=0, \\
i=1,2, \ldots, \mathrm{m}, \mathrm{j}=1,2, \ldots, \theta-1,
\end{gathered}
$$

where the left side of the equation is the discrete analogue of the Laplacian $\frac{\partial^{2} z}{\partial x^{2}}+\frac{\partial^{2} z}{\partial y^{2}}$ of brightness function $z=z(\mathrm{x}, y)$, and $z_{i, j}$ is an image brightness at the point $(i, j), u_{i, j}, v_{i, j}$ are the control parameters, $u_{i, j}$ is a control parameter of the chasing player, $v_{i, j}$ is a control parameter of the evading one. Without loss of generality, it is convenient to consider $(\operatorname{see}(*))$ that if either $i=0$, or $i=m+1$, or $j=0$, or $j=\theta$, then $z_{i, j}=0$, i.e. the image is bordered with pixels of zero values of brightness. The chase problem is posed as follow. The chasing player can alter the solutions of the equation $(*) z_{i, j}$ within the changes, $u_{i, j}$. An evading player can change the brightness of the image using its operations $v_{i, j}$ as well. We will study the problem of a chasing player, the chase problem. A chase is complete if $z_{i, j} \quad$ satisfy the condition: $\delta \leq z_{i, j} \leq \delta+\varepsilon, i_{0} \leq i \leq i_{1}, j_{0} \leq j \leq j_{1} \quad, \quad$ where $1 \leq i_{0}, i_{1} \leq m, 1 \leq \mathrm{j}_{0}, j_{1} \leq \theta-1$ for some pre-defined $\delta>0, \varepsilon>0$. This means that the value of brightness levels of the image $z_{i, j}$ in predetermined pixels was in a segment, which are managed by a chasing player. A chasing player wants to finish the game as soon as possible, and the evading one, generally speaking, block to him.

\section{Methodology}

Using the boundary conditions at $i=1,2, \ldots, m$ from (*), we obtain the system

$$
\begin{aligned}
& -4 z_{1, j}+z_{0, j}+z_{2, j}+z_{1, j-1}+z_{1, j+1}=-u_{1, j}+v_{1, j}, \\
& -4 z_{2, j}+z_{1, j}+z_{3, j}+z_{2, j-1}+z_{2, j+1}=-u_{2, j}+v_{2, j}, \\
& -4 z_{i, j}+z_{i-1, j}+z_{i+1, j}+z_{i, j-1}+z_{i, j+1}=-u_{i, j}+v_{i, j}, \\
& -4 z_{\mathrm{m}-1, j}+z_{\mathrm{m}-2, j}+z_{\mathrm{m}, j}+z_{\mathrm{m}-1, j-1}+z_{\mathrm{m}-1, j+1}= \\
& =-u_{\mathrm{m}-1, j}+v_{\mathrm{m}-1, j}, \\
& -4 z_{\mathrm{m}, j}+z_{m-1, j}+z_{m+1, j}+z_{\mathrm{m}, j-1}+z_{\mathrm{m}, j+1}= \\
& =-u_{\mathrm{m}, j}+v_{\mathrm{m}, j} .
\end{aligned}
$$

Indicating

$z_{j}=\left(z_{1, j}, z_{2, j}, \ldots, z_{m, j}\right)^{\mathrm{T}}, u_{j}=\left(\mathrm{u}_{1, j}, \mathrm{u}_{2, j}, \ldots, \mathrm{u}_{m, j}\right)^{\mathrm{T}}$,

$v_{j}=\left(v_{1, j}, v_{2, j}, \ldots, v_{m, j}\right)^{\mathrm{T}}$ 
we have

$$
\begin{gathered}
-z_{j-1}+C z_{j}-z_{j+1}=u_{j}-v_{j}, 1 \leq j \leq \theta-1, \\
z_{0}=0, z_{\theta}=0
\end{gathered}
$$

where $z_{j} \in R^{m}$ and $u_{j}$ is control parameter of a chasing player; $v_{j}$ is control parameter of evader: $u_{j} \in R^{m}, v_{j} \in R^{m} \quad$ components, which satisfies the condition $\left|u_{i, j}\right| \leq \rho,\left|v_{i, j}\right| \leq \sigma, \sigma<\rho$, and $\mathrm{C}$ is a square matrix.

The purpose of this work is the study of game problems of chase for the brightness levels of digital image described by discrete quasi-linear second-order equations. We obtained sufficient conditions in this class of discrete games for the possibility to finish the chase, when the position of the object specified in the boundary points.

Chebyshev's polynoms of the second sort [5], [6] are applied to the solution of this task and ideas of the first and second methods of Pontryagin L.S. are used.

\section{Results}

Instead of games $(* *)$ we will consider a more general discrete game, in which the movement of the point $z$ of m-dimensional Euclidean space $R^{m}$ is described by equations

$$
\begin{gathered}
-z_{j-1}+C z_{j}-z_{j+1}=f_{j}\left(u_{j}, v_{j}\right), 1 \leq j \leq \theta-1, \\
z_{0}=\phi_{0}, z_{\theta}=\phi_{\theta}
\end{gathered}
$$

where $\mathrm{j}$ is the step number, $\mathrm{C}$ is a constant square matrix mxm, $u, v$ are control parameters, $u$ is a chasing parameter, $v$ is a evading parameter, $u_{j} \in P_{j} \subset R^{p}, v_{j} \in Q_{j} \subset R^{q}, P_{j}$ and $Q_{j}$ are non-empty sets. The $u$ parameter is selected as a sequence $u=u()=.\left(\mathrm{u}_{1}, \mathrm{u}_{2}, \ldots, \mathrm{u}_{\theta-1}\right), \mathrm{u}_{j} \in P_{j}, j=1,2, \ldots, \theta-1$,

the $v$ parameter does as a sequence $v=v()=.\left(v_{1}, v_{2}, \ldots, v_{\theta-1}\right), v_{j} \in Q_{j}, j=1,2, \ldots, \theta-1, f_{j}$ is a given function, are displayed $R^{p} \times R^{q}$ in $R^{m}$. In addition, terminal set $M$ is allocated in the $R^{m}$. The purpose of chasing player to bring $z_{j}$ at $M$ set. An evading player is trying to prevent this.

Theory 1. $M=M_{0}+M_{1}$, where $M_{0}$ is a linear subspace of $R^{m} ; M_{1}$ is a subset of the subspace $L$, the orthogonal complement of $M_{0}$ and $R^{m}$. We indicate operation of orthogonal projection from $R^{m}$ on $L$. [4] Let $M_{1,1}+M_{1,2}=M_{1}$ and
$W_{1,1}(n)=$

$=-M_{11}+\sum_{k=1}^{n-1} \bigcap_{v_{k} \in Q_{k}} \Pi U_{\theta-1}^{-1}\left(\frac{1}{2} C\right) U_{\theta-n-1}\left(\frac{1}{2} C\right) U_{k-1}\left(\frac{1}{2} C\right) f_{k}\left(P_{k}, v_{k}\right)$

$W_{1,2}(n)=$

$=-M_{1,2}+\sum_{k=n}^{\theta-1} \bigcap_{v_{k} \in Q_{k}} \Pi U_{\theta-1}^{-1}\left(\frac{1}{2} C\right) U_{n-1}\left(\frac{1}{2} C\right) U_{\theta-k-1}\left(\frac{1}{2} C\right) f_{k}\left(P_{k}, v_{k}\right)$,

here the $U_{n}\left(\frac{1}{2} C\right)$ is Chebyshev's matrix polynomial.

Theory 2. Let there exist such $n=n_{0} \leq \theta-1$, that

$$
\begin{aligned}
& -\Pi\left[U_{\theta-1}^{-1}\left(\frac{1}{2} C\right) U_{\theta-n_{0}-1}\left(\frac{1}{2} C\right) z_{0}\right] \in W_{1,1}\left(n_{0}\right) \text { and } \\
& -\Pi\left[U_{\theta-1}^{-1}\left(\frac{1}{2} C\right) U_{n_{0}-1}\left(\frac{1}{2} C\right) z_{\theta}\right] \in W_{1,2}\left(n_{0}\right) .
\end{aligned}
$$

Theorem 1. If the Theories 1, 2 are executing, then at the games (1), (2) it is possible the finish of chase in $N\left(z_{0}, z_{\theta}\right) \leq n_{0}$ steps from the "boundary" point $\left(z_{0}, z_{\theta}\right)$.

Let $1 \leq n \leq \theta-1, W_{2,1}(0)=-M_{1,1}, W_{2,2}=-M_{1,2}$, $W_{2,1}(n)=$

$=\bigcap_{v_{k} \in Q_{k}}\left[W_{2,1}(n-1)+\Pi U_{\theta-1}^{-1}\left(\frac{1}{2} C\right) U_{\theta-n-1}\left(\frac{1}{2} C\right) U_{k-1}\left(\frac{1}{2} C\right) f_{k}\left(P_{k}, v_{k}\right)\right]$ , $1 \leq k \leq n-1$,

$W_{2,2}(n)=$

$=\bigcap_{v_{k} \in Q_{k}}\left[W_{2,2}(n-1)+\Pi U_{\theta-1}^{-1}\left(\frac{1}{2} C\right) U_{n-1}\left(\frac{1}{2} C\right) U_{\theta-k-1}\left(\frac{1}{2} C\right) f_{k}\left(P_{k}, v_{k}\right)\right]$ ,$n \leq k \leq \theta-1$.

Theory 3. Let there exist such $n=n_{0} \leq \theta-1$, that

$$
\begin{array}{r}
-\Pi\left[U_{\theta-1}^{-1}\left(\frac{1}{2} C\right) U_{\theta-n_{0}-1}\left(\frac{1}{2} C\right) z_{0}\right] \in W_{2,1}\left(n_{0}\right) \text { and } \\
-\Pi\left[U_{\theta-1}^{-1}\left(\frac{1}{2} C\right) U_{n_{0}-1}\left(\frac{1}{2} C\right) z_{\theta}\right] \in W_{2,2}\left(n_{0}\right) .
\end{array}
$$

Theorem 2. If the Theory 3 is executing, then at the games (1), (2) it is possible the finish of chase in $N\left(z_{0}, z_{\theta}\right) \leq n_{0}$ steps from the "boundary" point $\left(z_{0}, z_{\theta}\right)$.

Let $\alpha_{n}(\cdot)=\left\{\alpha_{1}, \alpha_{2}, \ldots, \alpha_{n-1}: \alpha_{k} \geq 0, \sum_{k=1}^{n-1} \alpha(k)=1\right\}$,

$\beta_{n}(\cdot)=\left\{\beta_{n}, \beta_{n+1}, \ldots, \beta_{\theta-1}: \beta_{k} \geq 0, \sum_{k=n}^{\theta-1} \beta(k)=1\right\}$ and 
$W_{1}\left(\alpha_{n}(\cdot)\right)=$

$=\sum_{k=1}^{n-1} \bigcap_{v_{k} \in Q_{k}}\left[\alpha_{k} M_{1,1}+\Pi U_{\theta-1}^{-1}\left(\frac{1}{2} C\right) U_{\theta-n-1}\left(\frac{1}{2} C\right) U_{k-1}\left(\frac{1}{2} C\right) f_{k}\left(P_{k}, v_{k}\right)\right]^{\prime}$

$W_{2}\left(\beta_{n}(\cdot)\right)=$

$=\sum_{k=n}^{\theta-1} \bigcap_{v_{k} \in Q_{K}}\left[\beta_{k} M_{1,2}+\Pi U_{\theta-1}^{-1}\left(\frac{1}{2} C\right) U_{n-1}\left(\frac{1}{2} C\right) U_{\theta-k-1}\left(\frac{1}{2} C\right) f_{k}\left(P_{k}, v_{k}\right)\right]$

Let $W_{3,1}(0)=M_{1,1}, \quad W_{3,1}(n)=\underset{\alpha_{k}(\cdot)}{U} W_{1}\left(\alpha_{k}(\cdot)\right) ，$ $1 \leq k \leq n-1$

$$
\begin{gathered}
W_{3,2}(0)=M_{1,2}, \quad W_{3,2}(n)=\underset{\beta_{k}(\cdot)}{U} W_{1}\left(\beta_{k}(\cdot)\right) \\
1 \leq k \leq \theta-1 .
\end{gathered},
$$

Theory 4. Let there exist such $n=n_{0} \leq \theta-1$, that

$$
\begin{aligned}
& -\Pi\left[U_{\theta-1}^{-1}\left(\frac{1}{2} C\right) U_{\theta-n_{0}-1}\left(\frac{1}{2} C\right) z_{0}\right] \in W_{3,1}\left(n_{0}\right) \\
& -\Pi\left[U_{\theta-1}^{-1}\left(\frac{1}{2} C\right) U_{n_{0}-1}\left(\frac{1}{2} C\right) z_{0}\right] \in W_{3,1}\left(n_{0}\right) .
\end{aligned}
$$

Theorem 3. If the Theory 4 is executing, then at the games (1), (2) it is possible the finish of chase in $N\left(z_{0}, z_{\theta}\right) \leq n_{0}$ steps from the "boundary" point $\left(z_{0}, z_{\theta}\right)$.

\section{Discussion}

Proof of the theorem 1. From (3) and (4) follows existence of such

$$
a(k) \in \begin{cases}\bigcap_{v(k) \in Q} \Pi U_{\theta-1}^{-1}\left(\frac{1}{2} C\right) U_{\theta-n_{0}-1}\left(\frac{1}{2} C\right) U_{k-1}\left(\frac{1}{2} C\right)(P-v(k)), & 1 \leq k \leq n_{0}-1, \\ \bigcap_{v(k) \in Q} \Pi U_{\theta-1}^{-1}\left(\frac{1}{2} C\right) U_{n_{0}-1}\left(\frac{1}{2} C\right) U_{\theta-k-1}\left(\frac{1}{2} C\right)(P-v(k)), & n_{0} \leq k \leq \theta-1,\end{cases}
$$

$$
b_{1} \in M_{1,1}, b_{2} \in M_{1,2} \text {, that }
$$

$$
\begin{aligned}
& -\Pi\left[U_{\theta-1}^{-1}\left(\frac{1}{2} C\right) U_{\theta-n_{0}-1}\left(\frac{1}{2} C\right) z_{0}\right]=\sum_{k=1}^{n_{0}-1} a(k)-b_{1}, \\
& -\Pi\left[U_{\theta-1}^{-1}\left(\frac{1}{2} C\right) U_{n_{0}-1}\left(\frac{1}{2} C\right) z_{\theta}\right]=\sum_{k=n_{0}}^{\theta-1} a(k)-b_{2} .
\end{aligned}
$$

Let $v=\bar{v}(k), \quad 1 \leq k \leq \theta-1 \quad-\quad$ any admissible management of the running-away player; management of the pursuing player $u=\bar{u}(k)$ we will construct as the solution of the following equation

$$
a(k)=\left\{\begin{array}{c}
\Pi U_{\theta-1}^{-1}\left(\frac{1}{2} C\right) U_{\theta-n_{0}-1}\left(\frac{1}{2} C\right) U_{k-1}\left(\frac{1}{2} C\right)(\bar{u}(k)-\bar{v}(k)), \\
1 \leq k \leq n_{0}-1 ; \\
\Pi U_{\theta-1}^{-1}\left(\frac{1}{2} C\right) U_{n_{0}-1}\left(\frac{1}{2} C\right) U_{\theta-k-1}\left(\frac{1}{2} C\right)(\bar{u}(k)-\bar{v}(k)) .
\end{array}\right.
$$

It is clear, that these equations have decisions on the choice $a(k)$, as $\bar{v}(k) \in Q$ and $\bar{u}(k) \in P$. Substitute $v=\bar{v}_{k}=\bar{v}(k)$ and $u=\bar{u}_{k}=\bar{u}(k)$ in (1) and applying a formula (3), we receive

$z\left(n_{0}\right)=$

$U_{\theta-1}^{-1}\left(\frac{1}{2} C\right) U_{\theta-n_{0}-1}\left(\frac{1}{2} C\right)\left[z_{0}+\sum_{k=1}^{n_{0}-1} U_{k-1}\left(\frac{1}{2} C\right)\left(\bar{u}_{k}-\bar{v}_{k}\right)\right]+$

$+U_{\theta-1}^{-1}\left(\frac{1}{2} C\right) U_{n_{0}-1}\left(\frac{1}{2} C\right)\left[z_{0}+\sum_{k=n_{0}}^{\theta-1} U_{\theta-k-1}\left(\frac{1}{2} C\right)\left(\bar{u}_{k}-\bar{v}_{k}\right)\right]$

From this, applying, to both parts of equality the operator of design $\Pi$ and from equality (5), (6), we have

$\Pi z\left(n_{0}\right)=$

$\Pi\left[U_{\theta-1}^{-1}\left(\frac{1}{2} C\right) U_{\theta-n_{0}-1}\left(\frac{1}{2} C\right) z_{0}+\sum_{k=1}^{n_{0}-1} U_{\theta-1}^{-1}\left(\frac{1}{2} C\right) U_{\theta-n_{0}-1}\left(\frac{1}{2} C\right)\right.$.

$\left.U_{k-1}\left(\frac{1}{2} C\right)\left(\bar{u}_{k}-\bar{v}_{k}\right)\right]+\Pi\left[U_{\theta-1}^{-1}\left(\frac{1}{2} C\right) U_{n_{0}-1}\left(\frac{1}{2} C\right) z_{\theta}+\right.$ $\left.+\sum_{k=n_{0}}^{\theta-1} U_{\theta-1}^{-1}\left(\frac{1}{2} C\right) U_{n_{0}-1}\left(\frac{1}{2} C\right) U_{\theta-k-1}\left(\frac{1}{2} C\right)\left(\bar{u}_{k}-\bar{v}_{k}\right)\right]=$ $=\Pi\left[U_{\theta-1}^{-1}\left(\frac{1}{2} C\right) U_{\theta-n_{0}-1}\left(\frac{1}{2} C\right) z_{0}\right]+\sum_{k=1}^{n_{0}-1} a(k)+$ $+\Pi\left[U_{\theta-1}^{-1}\left(\frac{1}{2} C\right) U_{n_{0}-1}\left(\frac{1}{2} C\right) z_{\theta}\right]+\sum_{k=n_{0}}^{\theta-1} a(k)$ $=\Pi\left[U_{\theta-1}^{-1}\left(\frac{1}{2} C\right) U_{\theta-n_{0}-1}\left(\frac{1}{2} C\right) z_{0}\right]-$ $-\Pi\left[U_{\theta-1}^{-1}\left(\frac{1}{2} C\right) U_{\theta-n_{0}-1}\left(\frac{1}{2} C\right) z_{0}\right]+b_{1}$ $+\Pi\left[U_{\theta-1}^{-1}\left(\frac{1}{2} C\right) U_{n_{0}-1}\left(\frac{1}{2} C\right) z_{\theta}\right]-$ $-\Pi\left[U_{\theta-1}^{-1}\left(\frac{1}{2} C\right) U_{n_{0}-1}\left(\frac{1}{2} C\right) z_{0}\right]+b_{2}=$. $=b_{1}+b_{2} \in M_{1,1}+M_{1,2}=M_{1}$

From this inclusion we will receive that $\Pi z\left(n_{0}\right) \in M_{1}$ and, mean, $z\left(n_{0}\right) \in M$. What should be proofed.

Theorem 1 is similar proofs as the theorem 2, 3 proofs. 


\section{Conclusions}

Thus, summarizing the results, we conclude that the discrete games of chasing (1), (2) describes the simplistic manageable process of digital image processing. In Theorems 1-3 we obtained sufficient conditions for the solutions of the corresponding problems (1), (2). Partially apply Theorems 1-3 to the model example we found that using the management in specified area, you could define a certain level of brightness in a digital image in case of presence of the player, which prevented this transition.

\section{REFERENCES}

[1] R. Gonzalez, R. Woods, Digital Image Processing. - M.,
Technosphere, 2005 - $1072 \mathrm{p}$.

[2] V.A Sadovnichy, J. Antonio, O.R Musin, O.V Seleznev, V.M Staroverov. Structural lines and critical points of the digital image // Selected topics of mathematics, mechanics and their applications. M.: Publishing House of Moscow. University Press, 1999., p 438-462.

[3] Y.I Monich, V.V Starovoytov. Quality Assessment for the analysis of digital

[4] Images // Artificial intelligence. Minsk. - 2008. - № 4. p.376- 386 .

[5] L.S Pontryagin. Linear differential games of pursuit // Mat. Collection, 1980, T. 112, number 3, p. 307-330.

[6] Samarski A.A., Nikolaev E.S. Methods of the solution of the net equations. - M.: Science, 1978, 570 p.

[7] Pashkovsky S. Computing applications of polynomials and Chebyshev's ranks. - M.: Science, 1983, 487 p. 